\title{
Robust Subgraph Generation Improves Abstract Meaning Representation Parsing
}

\author{
Keenon Werling \\ Stanford University \\ keenonestanford. edu
}

\author{
Gabor Angeli \\ Stanford University \\ angeliestanford.edu
}

\author{
Christopher D. Manning \\ Stanford University \\ manningestanford. edu
}

\begin{abstract}
The Abstract Meaning Representation (AMR) is a representation for opendomain rich semantics, with potential use in fields like event extraction and machine translation. Node generation, typically done using a simple dictionary lookup, is currently an important limiting factor in AMR parsing. We propose a small set of actions that derive AMR subgraphs by transformations on spans of text, which allows for more robust learning of this stage. Our set of construction actions generalize better than the previous approach, and can be learned with a simple classifier. We improve on the previous state-of-the-art result for AMR parsing, boosting end-to-end performance by $3 F_{1}$ on both the LDC2013E117 and LDC2014T12 datasets.
\end{abstract}

\section{Introduction}

The Abstract Meaning Representation (AMR) (Banarescu et al., 2013) is a rich, graph-based language for expressing semantics over a broad domain. The formalism is backed by a large datalabeling effort, and it holds promise for enabling a new breed of natural language applications ranging from semantically aware MT to rich broaddomain QA over text-based knowledge bases. Figure 1 shows an example AMR for "he gleefully ran to his dog Rover," and we give a brief introduction to AMR in Section 2. This paper focuses on AMR parsing, the task of mapping a natural language sentence into an AMR graph.

We follow previous work (Flanigan et al., 2014) in dividing AMR parsing into two steps. The first step is concept identification, which generates AMR nodes from text, and which we'll refer to as $N E R++($ Section 3.1). The second step is relation

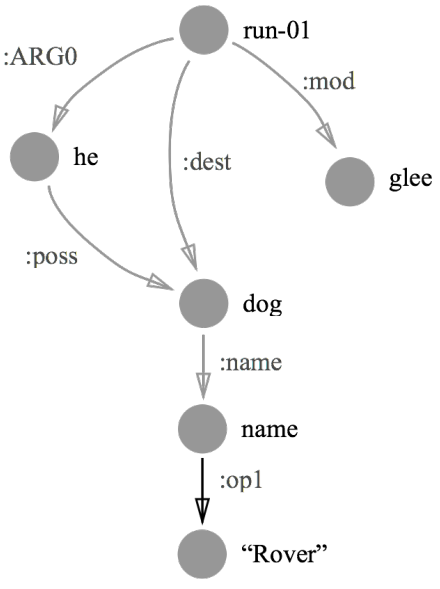

Figure 1: The AMR graph for He gleefully ran to his dog Rover. We show that improving the generation of low level subgraphs (e.g., Rover generating name $\stackrel{: o p 1}{\longrightarrow}$ "Rover") significantly improves end-to-end performance.

identification, which adds arcs to link these nodes into a fully connected AMR graph, which we'll call $S R L++$ (Section 3.2).

We observe that SRL++ is not the hard part of AMR parsing; rather, much of the difficulty in AMR is generating high accuracy concept subgraphs from the NER++ component. For example, when the existing AMR parser JAMR (Flanigan et al., 2014) is given a gold NER++ output, and must only perform SRL++ over given subgraphs it scores $80 \mathrm{~F}_{1}$ - nearly the inter-annotator agreement of $83 \mathrm{~F}_{1}$, and far higher than its end to end accuracy of $59 \mathrm{~F}_{1}$.

SRL++ within AMR is relatively easy given a perfect NER++ output, because so much pressure is put on the output of NER++ to carry meaningful information. For example, there's a strong typecheck feature for the existence and type of any arc just by looking at its end-points, and syntactic dependency features are very informative for removing any remaining ambiguity. If a system is con- 


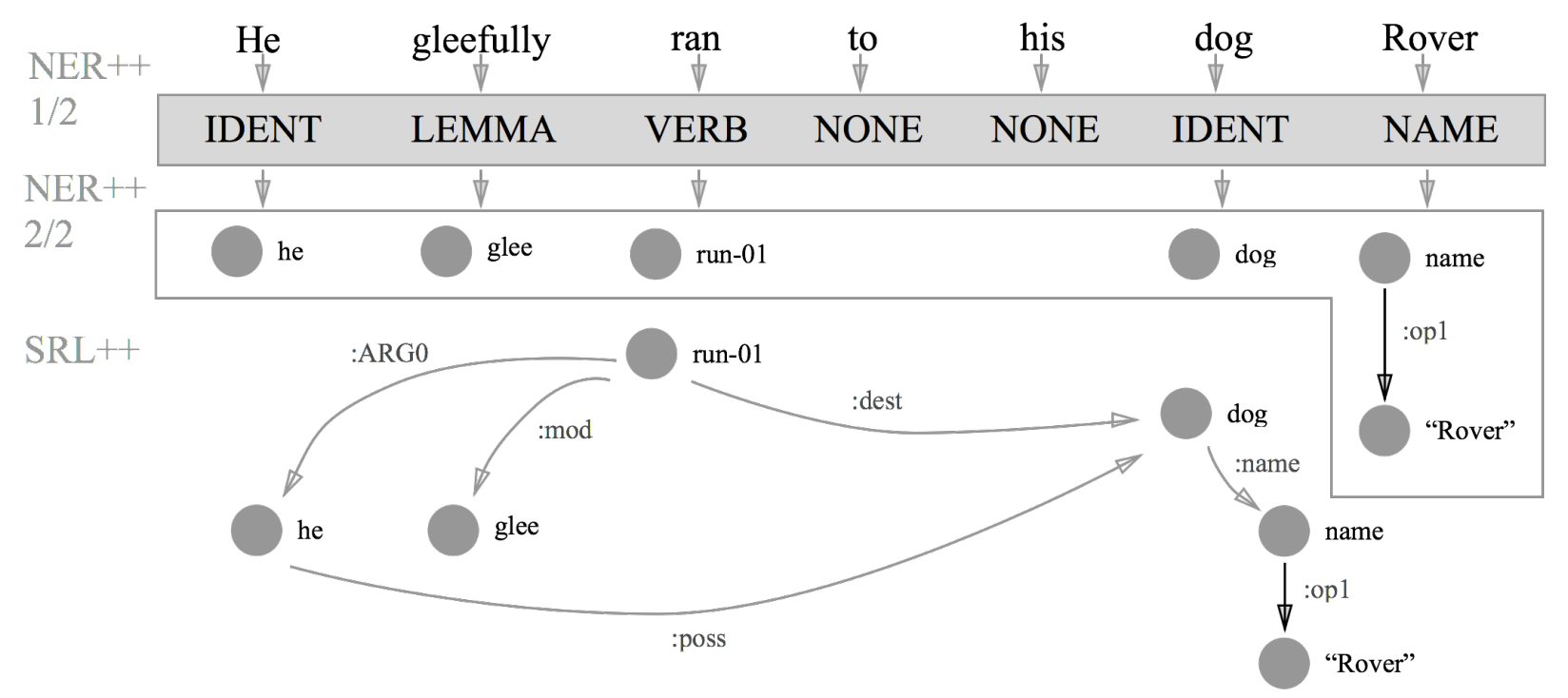

Figure 2: A graphical explanation of our method. We represent the derivation process for He gleefully ran to his dog Rover. First the tokens in the sentence are labeled with derivation actions, then these actions are used to generate AMR subgraphs, which are then stitched together to form a coherent whole.

sidering how to link the node run-01 in Figure 1. the verb-sense frame for "run-01" leaves very little uncertainty for what we could assign as an $A R G O$ arc. It must be a noun, which leaves either he or dog, and this is easily decided in favor of he by looking for an nsubj arc in the dependency parse.

The primary contribution of this paper is a novel approach to the NER++ task, illustrated in Figure 2. We notice that the subgraphs aligned to lexical items can often be generated from a small set of generative actions which generalize across tokens. For example, most verbs generate an AMR node corresponding to the verb sense of the appropriate PropBank frame - e.g., run generates run-01 in Figure 1. This allows us to frame the NER++ task as the task of classifying one of a small number of actions for each token, rather than choosing a specific AMR subgraph for every token in the sentence.

Our approach to the end-to-end AMR parsing task is therefore as follows: we define an action space for generating AMR concepts, and create a classifier for classifying lexical items into one of these actions (Section 4). This classifier is trained from automatically generated alignments between the gold AMR trees and their associated sentences (Section 5), using an objective which favors alignment mistakes which are least harmful to the NER++ component. Finally, the concept subgraphs are combined into a coherent AMR parse using the maximum spanning connected subgraph algorithm of Flanigan et al. (2014).

We show that our approach provides a large boost to recall over previous approaches, and that end to end performance is improved from 59 to 62 smatch (an $\mathrm{F}_{1}$ measure of correct AMR arcs; see Cai and Knight (2013)) when incorporated into the SRL++ parser of Flanigan et al. (2014). When evaluating the performance of our action classifier in isolation, we obtain an action classification accuracy of $84.1 \%$.

\section{The AMR Formalism}

AMR is a language for expressing semantics as a rooted, directed, and potentially cyclic graph, where nodes represent concepts and arcs are relationships between concepts. AMR is based on neo-Davidsonian semantics, (Davidson, 1967; Parsons, 1990). The nodes (concepts) in an AMR graph do not have to be explicitly grounded in the source sentence, and while such an alignment is often generated to train AMR parsers, it is not provided in the training corpora. The semantics of nodes can represent lexical items (e.g., $d o g$ ), sense tagged lexical items (e.g., run-01), type markers (e.g., date-entity), and a host of other phenomena.

The edges (relationships) in AMR describe one of a number of semantic relationships between concepts. The most salient of these is semantic role labels, such as the $A R G 0$ and destination arcs in Figure 2. However, often these arcs define 


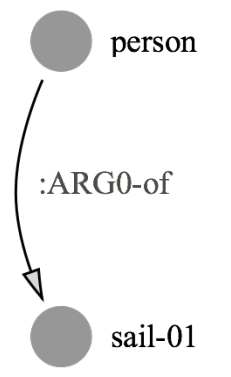

Figure 3: AMR representation of the word sailor, which is notable for breaking the word up into a self-contained multi-node unit unpacking the derivational morphology of the word.

a semantics more akin to syntactic dependencies (e.g., mod standing in for adjective and adverbial modification), or take on domain-specific meaning (e.g., the month, day, and year arcs of a dateentity).

To introduce AMR and its notation in more detail, we'll unpack the translation of the sentence "he gleefully ran to his dog Rover." We show in Figure 1 the interpretation of this sentence as an AMR graph.

The root node of the graph is labeled run-01, corresponding to the PropBank (Palmer et al., 2005) definition of the verb ran. run-01 has an outgoing $A R G O$ arc to a node $h e$, with the usual PropBank semantics. The outgoing mod edge from run-01 to glee takes a general purpose semantics corresponding to adjective, adverbial, or other modification of the governor by the dependent. We note that run-01 has a destination arc to $d o g$. The label for destination is taken from a finite set of special arc sense tags similar to the preposition senses found in (Srikumar, 2013). The last portion of the figure parses $d o g$ to a node which serves as a type marker similar to named entity types, and Rover into the larger subgraph indicating a concept with name "Rover."

\subsection{AMR Subgraphs}

The mapping from tokens of a sentence to AMR nodes is not one-to-one. A single token or span of tokens can generate a subgraph of AMR consisting of multiple nodes. These subgraphs can logically be considered the expression of a single concept, and are useful to treat as such (e.g., see Section 3.1.

Many of these multi-node subgraphs capture structured data such as time expressions, as in Fig-

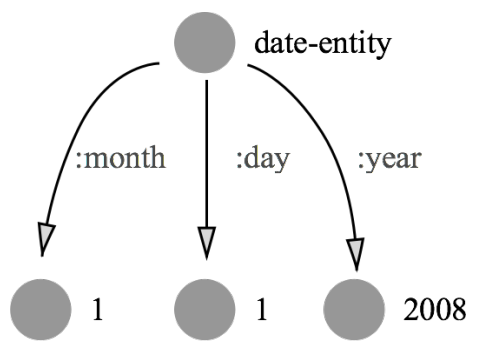

Figure 4: AMR representation of the span January 1, 2008, an example of how AMR can represent structured data by creating additional nodes such as date-entity to signify the presence of special structure.

ure 4 . In this example, a date-entity node is created to signify that this cluster of nodes is part of a structured sub-component representing a date, where the nodes and arcs within the component have specific semantics. This illustrates a broader recurring pattern in AMR: an artificial node may, based on its title, have expected children with special semantics. A particularly salient example of this pattern is the name node (see "Rover" in Figure 1) which signifies that all outgoing arcs with label op comprise the tokens of a name object.

The ability to decouple the meaning representation of a lexical item from its surface form allows for rich semantic interpretations of certain concepts in a sentence. For example, the token sailor is represented in Figure 3 by a concept graph representing a person who performs the action sail01. Whereas often the AMR node aligned to a span of text is a straightforward function of the text, these cases remain difficult to capture in a principled way beyond memorizing mappings between tokens and subgraphs.

\section{Task Decomposition}

To the best of our knowledge, the JAMR parser is the only published end-to-end AMR parser at the time of publication. An important insight in JAMR is that AMR parsing can be broken into two distinct tasks: (1) NER++ (concept identification): the task of interpreting what entities are being referred to in the text, realized by generating the best AMR subgraphs for a given set of tokens, and (2) SRL++ (relation identification): the task of discovering what relationships exist between entities, realized by taking the disjoint subgraphs generated by NER++ and creating a fullyconnected graph. We describe both tasks in more 
detail below.

\subsection{NER++}

Much of the difficulty of parsing to AMR lies in generating local subgraphs representing the meaning of token spans. For instance, the formalism implicitly demands rich notions of NER, lemmatization, word sense disambiguation, number normalization, and temporal parsing; among others. To illustrate, a correct parse of the sentence in Figure 2 requires lemmatization (gleefully $\rightarrow$ glee), word sense tagging (run $\rightarrow$ run-01), and open domain NER (i.e., Rover), Furthermore, many of the generated subgraphs (e.g., sailor in Figure 3 ) have rich semantics beyond those produced by standard NLP systems.

Formally, NER++ is the task of generating a disjoint set of subgraphs representing the meanings of localized spans of words in the sentence. For NER++, JAMR uses a simple Viterbi sequence model to directly generate AMR-subgraphs from memorized mappings of text spans to subgraphs. This paper's main contribution, presented in Section 4 , is to make use of generative actions to generate these subgraphs, rather than appealing to a memorized mapping.

\subsection{SRL++}

The second stage of the AMR decomposition consists of generating a coherent graph from the set of disjoint subgraphs produced by NER++. Whereas NER++ produces subgraphs whose arcs encode domain-specific semantics (e.g., month), the arcs in SRL++ tend to have generally applicable semantics. For example, the many arcs encode conventional semantic roles (e.g., ARGO and destination in Figure 2), or a notion akin to syntactic dependencies (e.g., mod and poss in Figure 2). For SRL++, JAMR uses a variation of the maximum spanning connected graph algorithm augmented by dual decomposition to impose linguistically motivated constraints on a maximum likelihood objective.

\section{A Novel NER++ Method}

The training sets currently available for AMR are not large. To illustrate, $38 \%$ of the words in the LDC2014E113 dev set are unseen during training time. With training sets this small, memorizationbased approaches are extremely brittle. We remove much of the necessity to memorize map- pings in NER++ by partitioning the AMR subgraph search space in terms of the actions needed to derive a node from its aligned token. At test time we do a sequence labeling of input tokens with these actions, and then deterministically derive the AMR subgraphs from spans of tokens by applying the transformation decreed by their actions. We explain in Section 4.1 how exactly we manage this partition, and in Section 4.3 how we create training data from existing resources to setup and train an action-type classifier.

\subsection{Derivation actions}

We partition the AMR subgraph space into a set of 9 actions, each corresponding to an action that will be taken by the NER++ system if a token receives this classification.

IDENTITY This action handles the common case that the title of the node corresponding to a token is identical to the source token. To execute the action, we take the lowercased version of the token to be the title of the corresponding node.

NONE This action corresponds to ignoring this token, in the case that the node should not align to any corresponding AMR fragment.

VERB This action captures the verb-sense disambiguation feature of AMR. To execute on a token, we find the most similar verb in PropBank based on Jaro-Winkler distance, and adopt its most frequent sense. This serves as a reasonable baseline for word sense disambiguation, although of course accuracy would be expected to improve if a sophisticated system were incorporated.

VALUE This action interprets a token by its integer value. The AMR representation is sensitive to the difference between a node with a title of 5 (the integer value) and " 5 " or "five" - the string value. This is a rare action, but is nonetheless distinct from any of the other classes. We execute this action by extracting an integer value with a regex based number normalizer, and using the result as the title of the generated node.

LEMMA AMR often performs stemming and part-of-speech transformations on the source token in generating a node. For example, we get glee from gleefully. We capture this by a LEMMA action, which is executed by using the lemma of the source token as the generated node title. Note that this does not capture all lemmatizations, as 
there are often discrepancies between the lemma generated by the lemmatizer and the correct AMR lemma.

NAME AMR often references names with a special structured data type: the name construction. For example, Rover in Figure 1. We can capture this phenomenon on unseen names by attaching a created name node to the top of a span.

PERSON A variant of the NAME action, this action produces a subgraph identical to the NAME action, but adds a node person as a parent. This is, in effect, a name node with an implicit entity type of person. Due to discrepancies between the output of our named entity tagger and the richer AMR named entity ontology, we only apply this tag to the person named entity tag.

DATE The most frequent of the structured data type in the data, after name, is the date-entity construction (for an example see Figure 4). We deterministically take the output of SUTime (Chang and Manning, 2012) and convert it into the dateentity AMR representation.

DICT This class serves as a back-off for the other classes, implementing an approach similar to Flanigan et al. (2014). In particular, we memorize a simple mapping from spans of text (such as sailor) to their corresponding most frequently aligned AMR subgraphs in the training data (i.e., the graph in Figure 3). See Section 5 for details on the alignment process. At test time we can do a lookup in this dictionary for any element that gets labeled with a DICT action. If an entry is not found in the mapping, we back off to the second most probable class proposed by the classifier.

It is worth observing at this point that our actions derive much of their power from the similarity between English words and their AMR counterparts; creating an analogue of these actions for other languages remains an open problem.

\subsection{Action Reliability}

In many cases, multiple actions could yield the same subgraph when applied to a node. In this section we introduce a method for resolving this ambiguity based on comparing the reliability with which actions generate the correct subgraph, and discuss implications.

Even given a perfect action classification for a token, certain action executions can introduce

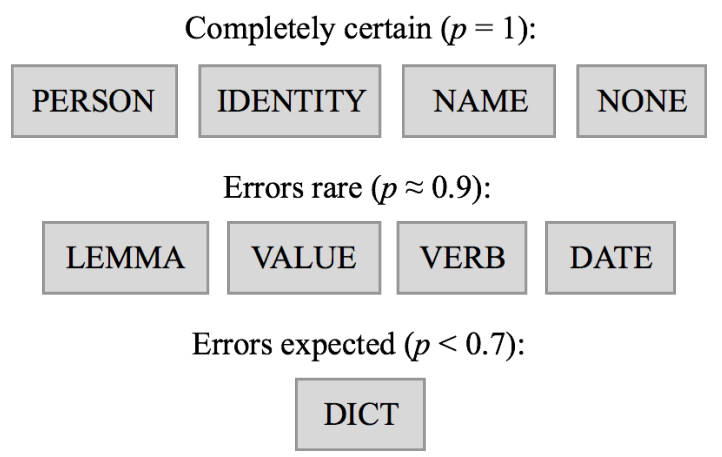

Figure 5: Reliability of each action. The top row are actions which are deterministic; the second row occasionally produce errors. DICT is the least preferred action, with a relatively high error rate.

errors. Some of our actions are entirely deterministic in their conversion from the word to the AMR subgraph (e.g., IDENTITY), but others are prone to making mistakes in this conversion (e.g., VERB, DICT). We define the notion of action reliability as the probability of deriving the correct node from a span of tokens, conditioned on having chosen the correct action.

To provide a concrete example, our dictionary lookup classifier predicts the correct AMR subgraph $67 \%$ of the time on the dev set. We therefore define the reliability of the DICT action as 0.67. In contrast to DICT, correctly labeling a node as IDENTITY, NAME, PERSON, and NONE have action reliability of 1.0, since there is no ambiguity in the node generation once one of those actions have been selected, and we are guaranteed to generate the correct node given the correct action.

We can therefore construct a hierarchy of reliability (Figure 5) - all else being equal, we prefer to generate actions from higher in the hierarchy, as they are more likely to produce the correct subgraph. This hierarchy is useful in resolving ambiguity throughout our system. During the creation of training data for our classifier (Section 4.3 from our aligner, when two actions could both generate the aligned AMR node we prefer the more reliable one. In turn, in our aligner we bias alignments towards those which generating more reliable action sequences as training data (see Section 5).

The primary benefit of this action-based NER++ approach is that we can reduce the usage of low reliability actions, like DICT. The approach taken in Flanigan et al. (2014) can be 


\begin{tabular}{l|rr} 
Action & \# Tokens & \% Total \\
\hline NONE & 41538 & 36.2 \\
DICT & 30027 & 26.1 \\
IDENTITY & 19034 & 16.6 \\
VERB & 11739 & 10.2 \\
LEMMA & 5029 & 4.5 \\
NAME & 4537 & 3.9 \\
DATE & 1418 & 1.1 \\
PERSON & 1336 & 1.1 \\
VALUE & 122 & 0.1
\end{tabular}

Table 1: Distribution of action types in the proxy section of the newswire section of the LDC2014T12 dataset, generated from automatically aligned data.

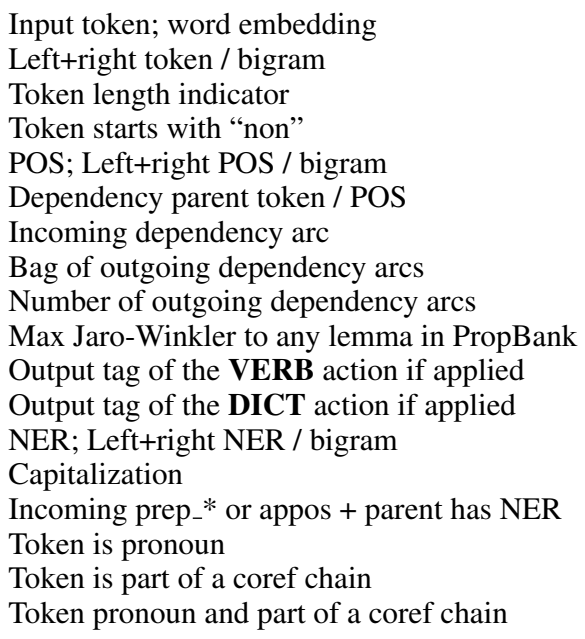

Table 2: The features for the NER++ maxent classifier.

thought of as equivalent to classifying every token as the DICT action.

We analyze the empirical distribution of actions in our automatically aligned corpus in Table 1 The cumulative frequency of the non-DICT actions is striking: we can generate $74 \%$ of the tokens with high reliability $(p \geq 0.9)$ actions. In this light, it is unsurprising that our results demonstrate a large gain in recall on the test set.

\subsection{Training the Action Classifier}

Given a set of AMR training data, in the form of (graph, sentence) pairs, we first induce alignments from the graph nodes to the sentence (see Section 5). Formally, for every node $n_{i}$ in the AMR graph, alignment gives us some token $s_{j}$ (at the $j$ th index in the sentence) that we believe generated the node $n_{i}$.
Then, for each action type, we can ask whether or not that action type is able to take token $s_{j}$ and correctly generate $n_{i}$. For concreteness, imagine the token $s_{j}$ is running, and the node $n_{i}$ has the title run-01. The two action types we find that are able to correctly generate this node are DICT and VERB. We choose the most reliable action type of those available (see Figure 5) to generate the observed node - in this case, VERB.

In cases where an AMR subgraph is generated from multiple tokens, we assign the action label to each token which generates the subgraph. Each of these tokens are added to the training set; at test time, we collapse sequences of adjacent identical action labels, and apply the action once to the resulting token span.

Inducing the most reliable action (according to the alignments) for every token in the training corpus provides a supervised training set for our action classifier, with some noise introduced by the automatically generated alignments. We then train a simple maxent classifier 1 to make action decisions at each node. At test time, the classifier takes as input a pair $\langle i, S\rangle$, where $i$ is the index of the token in the input sentence, and $S$ is a sequence tokens representing the source sentence. It then uses the features in Table 2 to predict the actions to take at that node.

\section{Automatic Alignment of Training Data}

AMR training data is in the form of bi-text, where we are given a set of (sentence, graph) pairs, with no explicit alignments between them. We would like to induce a mapping from each node in the AMR graph to the token it represents. It is perfectly possible for multiple nodes to align to the same token - this is the case with sailors, for instance.

It is not possible, within our framework, to represent a single node being sourced from multiple tokens. Note that a subgraph can consist of many individual nodes; in cases where a subgraph should align to multiple tokens, we generate an alignment from the subgraph's nodes to the associated tokens in the sentence. It is empirically very rare for a subgraph to have more nodes than the token span it should align to.

There have been two previous attempts at producing automatic AMR alignments. The first was

\footnotetext{
${ }^{1}$ A sequence model was tried and showed no improvement over a simple maxent classifier.
} 
published as a component of JAMR, and used a rule-based approach to perform alignments. This was shown to work well on the sample of 100 hand-labeled sentences used to develop the system. Pourdamghani et al. (2014) approached the alignment problem in the framework of the IBM alignment models. They rendered AMR graphs as text, and then used traditional machine translation alignment techniques to generate an alignment.

We propose a novel alignment method, since our decomposition of the AMR node generation process into a set of actions provides an additional objective for the aligner to optimize, in addition to the accuracy of the alignment itself. We would like to produce the most reliable sequence of actions for the NER++ model to train from, where reliable is taken in the sense defined in Section 4.2. To give an example, a sequence of all DICT actions could generate any AMR graph, but is very low reliability. A sequence of all IDENTITY actions could only generate one set of nodes, but does it with absolute certainty.

We formulate this objective as a Boolean LP problem. Let $\mathbf{Q}$ be a matrix in $\{0,1\}^{|\mathbf{N}| \times|\mathbf{S}|}$ of Boolean constrained variables, where $\mathbf{N}$ are the nodes in an AMR graph, and $\mathbf{S}$ are the tokens in the sentence. The meaning of $\mathbf{Q}_{i, j}=\mathbb{1}$ can be interpreted as node $n_{i}$ having being aligned to token $s_{j}$. Furthermore, let $\mathbf{V}$ be a matrix $\mathcal{T}^{|\mathbf{N}| \times|\mathbf{S}|}$, where $\mathcal{T}$ is the set of NER++ actions from Section 4 Each matrix element $\mathbf{V}_{i, j}$ is assigned the most reliable action which would generate node $n_{i}$ from token $s_{j}$. We would like to maximize the probability of the actions collectively generating a perfect set of nodes. This can be formulated linearly by maximizing the log-likelihood of the actions. Let the function $\operatorname{REL}(l)$ be the reliability of action $l$ (probability of generating intended node). Our objective can then be formulated as follows:

$$
\begin{array}{ll}
\max _{\mathbf{Q}} & \sum_{i, j} \mathbf{Q}_{i, j}\left[\log \left(\operatorname{REL}\left(\mathbf{V}_{i, j}\right)\right)+\alpha \mathcal{E}_{i, j}\right] \\
\text { s.t. } & \sum_{j} \mathbf{Q}_{i, j}=1 \quad \forall i \\
& \mathbf{Q}_{k, j}+\mathbf{Q}_{l, j} \leq 1 \quad \forall k, l, j ; n_{k} \leftrightarrow n_{l}
\end{array}
$$

where $\mathcal{E}$ is the Jaro-Winkler similarity between the title of the node $i$ and the token $j, \alpha$ is a hyperparameter (set to 0.8 in our experiments), and the operator $\leftrightarrow$ denotes that two nodes in the AMR graph are both not adjacent and do not have the same title.
The constraint (2), combined with the binary constraint on $\mathbf{Q}$, ensures that every node in the graph is aligned to exactly one token in the source sentence. The constraint (3) ensures that only adjacent nodes or nodes that share a title can refer to the same token.

The objective value penalizes alignments which map to the unreliable DICT tag, while rewarding alignments with high overlap between the title of the node and the token. Note that most incorrect alignments fall into the DICT class by default, as no other action could generate the correct AMR subgraph. Therefore, if there exists an alignment that would consume the token using another action, the optimization prefers that alignment. The Jaro-Winkler similarity term, in turn, serves as a tie-breaker between equally (un)reliable alignments.

There are many packages which can solve this Boolean LP efficiently. We used Gurobi (Gurobi Optimization, 2015). Given a matrix Q that maximizes our objective, we can decode our solved alignment as follows: for each $i$, align $n_{i}$ to the $j$ s.t. $\mathbf{Q}_{i, j}=1$. By our constraints, exactly one such $j$ must exist.

\section{Related Work}

Prior work in AMR and related formalisms include Jones et al. (2012), and Flanigan et al. (2014). Jones et al. (2012), motivated by applications in Machine Translation, proposed a graphical semantic meaning representation that predates AMR, but is intimately related. They propose a hyper-edge replacement grammar (HRG) approach to parsing into and out of this graphical semantic form. Flanigan et al. (2014) forms the basis of the approach of this paper. Their system introduces the two-stage approach we use: they implement a rule-based alignment to learn a mapping from tokens to subgraphs, and train a variant of a maximum spanning tree parser adapted to graphs and with additional constraints for their relation identifications (SRL++) component. Wang et al. (2015) uses a transition based algorithm to transform dependency trees into AMR parses. They achieve 64/62/63 P/R/F 1 with contributions roughly orthogonal to our own. Their transformation action set could be easily augmented by the robust subgraph generation we propose here, although we leave this to future work.

Beyond the connection of our work with Flani- 
gan et al. (2014), we note that the NER++ component of AMR encapsulates a number of lexical NLP tasks. These include named entity recognition (Nadeau and Sekine, 2007; Finkel et al., 2005), word sense disambiguation (Yarowsky, 1995: Banerjee and Pedersen, 2002), lemmatization, and a number of more domain specific tasks. For example, a full understanding of AMR requires normalizing temporal expressions (Verhagen et al., 2010; Strötgen and Gertz, 2010; Chang and Manning, 2012).

In turn, the SRL++ facet of AMR takes many insights from semantic role labeling (Gildea and Jurafsky, 2002; Punyakanok et al., 2004; Srikumar, 2013; Das et al., 2014) to capture the relations between verbs and their arguments. In addition, many of the arcs in AMR have nearly syntactic interpretations (e.g., mod for adjective/adverb modification, $o p$ for compound noun expressions). These are similar to representations used in syntactic dependency parsing (de Marneffe and Manning, 2008; McDonald et al., 2005; Buchholz and Marsi, 2006).

More generally, parsing to a semantic representation is has been explored in depth for when the representation is a logical form (Kate et al., 2005, Zettlemoyer and Collins, 2005: Liang et al., 2011). Recent work has applied semantic parsing techniques to representations beyond lambda calculus expressions. For example, work by Berant et al. (2014) parses text into a formal representation of a biological process. Hosseini et al. (2014) solves algebraic word problems by parsing them into a structured meaning representation. In contrast to these approaches, AMR attempts to capture open domain semantics over arbitrary text.

Interlingua (Mitamura et al., 1991; Carbonell et al., 1999; Levin et al., 1998) are an important inspiration for decoupling the semantics of the AMR language from the surface form of the text being parsed; although, AMR has a self-admitted English bias.

\section{Results}

We present improvements in end-to-end AMR parsing on two datasets using our NER++ component. Action type classifier accuracy on an automatically aligned corpus and alignment accuracy on a small hand-labeled corpus are also reported.

\begin{tabular}{l|l|llr} 
Dataset & System & $\mathbf{P}$ & $\mathbf{R}$ & $\mathbf{F}_{1}$ \\
\hline \multirow{2}{*}{$2014 \mathrm{~T} 12$} & JAMR & $\mathbf{6 7 . 1}$ & 53.2 & 59.3 \\
& Our System & 66.6 & $\mathbf{5 8 . 3}$ & $\mathbf{6 2 . 2}$ \\
\hline \multirow{2}{*}{$2013 \mathrm{E} 117$} & JAMR & $\mathbf{6 6 . 9}$ & 52.9 & 59.1 \\
& Our System & 65.9 & $\mathbf{5 9 . 0}$ & $\mathbf{6 2 . 3}$
\end{tabular}

Table 3: Results on two AMR datasets for JAMR and our NER++ embedded in the JAMR SRL++ component. Note that recall is consistently higher across both datasets, with only a small loss in precision.

\subsection{End-to-end AMR Parsing}

We evaluate our NER++ component in the context of end-to-end AMR parsing on two corpora: the newswire section of LDC2014T12 and the split given in Flanigan et al. (2014) of LDC2013E117, both consisting primarily of newswire. We compare two systems: the JAMR parser (Flanigan et al., 2014).2 and the JAMR SRL++ component with our NER++ approach.

AMR parsing accuracy is measured with a metric called smatch (Cai and Knight, 2013), which stands for "s(emantic) match." The metric is the $F_{1}$ of a best-match between triples implied by the target graph, and triples in the parsed graph - that is, the set of (parent, edge, child) triples in the graph.

Our results are given in Table 3 . We report much higher recall numbers on both datasets, with only small ( $\leq 1$ point) loss in precision. This is natural considering our approach. A better NER++ system allows for more correct AMR subgraphs to be generated - improving recall - but does not in itself necessarily improve the accuracy of the SRL++ system it is integrated in.

\subsection{Component Accuracy}

We evaluate our aligner on a small set of 100 handlabeled alignments, and evaluate our NER++ classifier on automatically generated alignments over the whole corpus,

On a hand-annotated dataset of 100 AMR parses from the LDC2014T12 corpus $3^{3}$ our aligner achieves an accuracy of 83.2. This is a measurement of the percentage of AMR nodes that are aligned to the correct token in their source sentence. Note that this is a different metric than the

\footnotetext{
${ }^{2}$ Available at https://github.com/jflanigan/ jamr

Our dataset is publicly available at http://nlp. stanford.edu/projects/amr
} 
precision/recall of prior work on alignments, and is based on both a different alignment dataset and subtly different alignment annotation scheme. In particular, we require that every AMR node aligns to some token in the sentence, which forces the system to always align nodes, even when unsure. A standard semantics and annotation guideline for AMR alignment is left for future work; our accuracy should be considered only an informal metric.

We find our informativeness-based alignment objective slightly improves end-to-end performance when compared to the rule-based approach of (Flanigan et al., 2014), improving $F_{1}$ by roughly 1 point $\left(64 / 59 / 61 \mathrm{P} / \mathrm{R} / \mathrm{F}_{1}\right.$ to $\left.65 / 59 / 62 \mathrm{P} / \mathrm{R} / \mathrm{F}_{1}\right)$.

On the automatic alignments over the LDC2014T12 corpus, our action classifier achieved a test accuracy of $\mathbf{0 . 8 4 1}$. The classifier's most common class of mistakes are incorrect DICT classifications. It is reassuring that some of these errors can be recovered from by the naïve dictionary lookup finding the correct mapping.

The DICT action lookup table achieved an accuracy of 0.67. This is particularly impressive given that our model moves many of the difficult semantic tasks onto the DICT tag, and that this lookup does not make use of any learning beyond a simple count of observed span to subgraph mappings.

\section{Conclusion}

We address a key challenge in AMR parsing: the task of generating subgraphs from lexical items in the sentence. We show that a simple classifier over actions which generate these subgraphs improves end-to-end recall for AMR parsing with only a small drop in precision, leading to an overall gain in $\mathrm{F}_{1}$. A clear direction of future work is improving the coverage of the defined actions. For example, a richer lemmatizer could shift the burden of lemmatizing unknown words into the AMR lemma semantics and away from the dictionary lookup component. We hope our decomposition provides a useful framework to guide future work in NER++ and AMR in general.

\section{Acknowledgments}

We thank the anonymous reviewers for their thoughtful feedback. Stanford University gratefully acknowledges the support of the Defense Advanced Research Projects Agency (DARPA) Deep Exploration and Filtering of Text (DEFT) Program under Air Force Research Laboratory (AFRL) contract no. FA8750-13-2-0040. Any opinions, findings, and conclusion or recommendations expressed in this material are those of the authors and do not necessarily reflect the view of the DARPA, AFRL, or the US government.

\section{References}

[Banarescu et al.2013] Laura Banarescu, Claire Bonial, Shu Cai, Madalina Georgescu, Kira Griffitt, Ulf Hermjakob, Kevin Knight, Philipp Koehn, Martha Palmer, and Nathan Schneider. 2013. Abstract meaning representation for sembanking. Proc. Linguistic Annotation Workshop.

[Banerjee and Pedersen2002] Satanjeev Banerjee and Ted Pedersen. 2002. An adapted Lesk algorithm for word sense disambiguation using wordnet. In Computational linguistics and intelligent text processing.

[Berant et al.2014] Jonathan Berant, Vivek Srikumar, Pei-Chun Chen, Brad Huang, Christopher D Manning, Abby Vander Linden, Brittany Harding, and Peter Clark. 2014. Modeling biological processes for reading comprehension. In Proc. EMNLP.

[Buchholz and Marsi2006] Sabine Buchholz and Erwin Marsi. 2006. CONLL-X shared task on multilingual dependency parsing. In Proceedings of the Tenth Conference on Computational Natural Language Learning, pages 149-164. Association for Computational Linguistics.

[Cai and Knight2013] Shu Cai and Kevin Knight. 2013. Smatch: an evaluation metric for semantic feature structures. In $A C L$ (2), pages 748-752.

[Carbonell et al.1999] Jaime G Carbonell, Teruko Mitamura, and Eric H Nyberg. 1999. The KANT perspective: A critique of pure transfer (and pure interlingua, pure statistics,...).

[Chang and Manning2012] Angel Chang and Chris Manning. 2012. SUTIME: a library for recognizing and normalizing time expressions. In Language Resources and Evaluation.

[Das et al.2014] Dipanjan Das, Desai Chen, André FT Martins, Nathan Schneider, and Noah A Smith. 2014. Frame-semantic parsing. Computational Linguistics, 40(1):9-56.

[Davidson1967] Donald Davidson. 1967. The logical form of action sentences. In Nicholas Rescher, editor, The Logic of Decision and Action, pages 81120. University of Pittsburgh Press, Pittsburgh, PA.

[de Marneffe and Manning2008] Marie-Catherine de Marneffe and Christopher D. Manning. 2008. The Stanford typed dependencies representation. In Coling 2008: Proceedings of the workshop on Cross-Framework and Cross-Domain Parser Evaluation. 
[Finkel et al.2005] Jenny Rose Finkel, Trond Grenager, and Christopher Manning. 2005. Incorporating non-local information into information extraction systems by Gibbs sampling. In $A C L$.

[Flanigan et al.2014] Jeffrey Flanigan, Sam Thomson, Jaime Carbonell, Chris Dyer, and Noah A Smith. 2014. A discriminative graph-based parser for the abstract meaning representation. In $A C L$.

[Gildea and Jurafsky2002] Daniel Gildea and Daniel Jurafsky. 2002. Automatic labeling of semantic roles. Computational linguistics, 28(3):245-288.

[Gurobi Optimization2015] Inc. Gurobi Optimization. 2015. Gurobi optimizer reference manual.

[Hosseini et al.2014] Mohammad Javad Hosseini, Hannaneh Hajishirzi, Oren Etzioni, and Nate Kushman. 2014. Learning to solve arithmetic word problems with verb categorization. In $E M N L P$.

[Jones et al.2012] Bevan Jones, Jacob Andreas, Daniel Bauer, Karl Moritz Hermann, and Kevin Knight. 2012. Semantics-based machine translation with hyperedge replacement grammars. In COLING, pages $1359-1376$.

[Kate et al.2005] Rohit J. Kate, Yuk Wah Wong, and Raymond J. Mooney. 2005. Learning to transform natural to formal languages. In $A A A I$, Pittsburgh, PA.

[Levin et al.1998] Lori S Levin, Donna Gates, Alon Lavie, and Alex Waibel. 1998. An interlingua based on domain actions for machine translation of taskoriented dialogues. In ICSLP, volume 98, pages 1155-1158.

[Liang et al.2011] P. Liang, M. I. Jordan, and D. Klein. 2011. Learning dependency-based compositional semantics. In $A C L$.

[McDonald et al.2005] Ryan McDonald, Koby Crammer, and Fernando Pereira. 2005. Online largemargin training of dependency parsers. In $A C L$, Morristown, NJ, USA.

[Mitamura et al.1991] Teruko Mitamura, Eric H Nyberg, and Jaime G Carbonell. 1991. An efficient interlingua translation system for multi-lingual document production. Proceedings of Machine Translation Summit III.

[Nadeau and Sekine2007] David Nadeau and Satoshi Sekine. 2007. A survey of named entity recognition and classification. Lingvisticae Investigationes, 30(1):3-26.

[Palmer et al.2005] Martha Palmer, Daniel Gildea, and Paul Kingsbury. 2005. The proposition bank: An annotated corpus of semantic roles. Computational linguistics, 31(1):71-106.

[Parsons1990] Terence Parsons. 1990. Events in the Semantics of English: A study in subatomic semantics. MIT Press, Cambridge, MA.
[Pourdamghani et al.2014] Nima Pourdamghani, Yang Gao, Ulf Hermjakob, and Kevin Knight. 2014. Aligning english strings with abstract meaning representation graphs. In EMNLP.

[Punyakanok et al.2004] Vasin Punyakanok, Dan Roth, Wen-tau Yih, and Dav Zimak. 2004. Semantic role labeling via integer linear programming inference. In Proceedings of the 20th international conference on Computational Linguistics, page 1346. Association for Computational Linguistics.

[Srikumar2013] Vivek Srikumar. 2013. The semantics of role labeling. Ph.D. thesis, University of Illinois at Urbana-Champaign.

[Strötgen and Gertz2010] Jannik Strötgen and Michael Gertz. 2010. Heideltime: High quality rule-based extraction and normalization of temporal expressions. In Proceedings of the 5th International Workshop on Semantic Evaluation, Sem-Eval.

[Verhagen et al.2010] Marc Verhagen, Roser Sauri, Tommaso Caselli, and James Pustejovsky. 2010. Semeval-2010 task 13: TempEval-2. In Proceedings of the 5th International Workshop on Semantic Evaluation, Uppsala, Sweden.

[Wang et al.2015] Chuan Wang, Nianwen Xue, and Sameer Pradhan. 2015. A transition-based algorithm for amr parsing. In NAACL-HLT.

[Yarowsky1995] David Yarowsky. 1995. Unsupervised word sense disambiguation rivaling supervised methods. In $A C L$.

[Zettlemoyer and Collins2005] Luke S. Zettlemoyer and Michael Collins. 2005. Learning to map sentences to logical form: Structured classification with probabilistic categorial grammars. In UAI. AUAI Press. 